\title{
Prevalence of pulmonary hypertension in pulmonary sarcoidosis: the first large European prospective study
}

\author{
To the Editor:
}

Sarcoidosis is a systemic disease of an unknown aetiology, in which noncaseating granulomas are formed in one or multiple organs, with pulmonary involvement in $>90 \%$ of the sarcoidosis patients [1]. Pulmonary hypertension $(\mathrm{PH})$, defined as a mean pulmonary artery pressure of $\geqslant 25 \mathrm{mmHg}$ by right heart catheterisation (RHC) [2], is a well recognised complication of sarcoidosis, associated with significant increase in mortality [3, 4]. Although the first case of PH in sarcoidosis was described in 1949 [5], the exact prevalence remains unclear. Only three studies have previously investigated the $\mathrm{PH}$ prevalence independently of suggestive symptoms and signs for $\mathrm{PH}$, resulting in prevalence rates of $5.7 \%, 14 \%$ and $20.8 \%$ [6-8]. In patients with complaints suggestive of $\mathrm{PH}$ or those awaiting lung transplant, rates of $\mathrm{PH}$ up to $79 \%$ have been reported [9-11]. Unfortunately, most studies are retrospective and have used an echocardiographic definition for $\mathrm{PH}$ (increased right ventricular systolic pressure (RVSP) of $\geqslant 40 \mathrm{mmHg}$ ), lacking RHC as gold standard.

A European Caucasian cohort has never been studied. The PULmonary hypertension in pulmonary SARcoidosis (PULSAR) study prospectively investigates the $\mathrm{PH}$ prevalence in patients with pulmonary sarcoidosis referred to a Dutch tertiary sarcoidosis centre (www.trialregister.nl; registration number NTR5295). This study was funded by ZonMW (The Netherlands Organisation for Health Research and Development).

Between August 2015 and October 2017, this cross-sectional study prospectively investigated the prevalence of $\mathrm{PH}$ in patients with histologically confirmed or confident clinical diagnosis of sarcoidosis. Patients with an age of $\geqslant 18$ years who were newly referred to the pulmonology department of the St Antonius Hospital Nieuwegein (the Netherlands), a tertiary centre for sarcoidosis and $\mathrm{PH}$, were asked for informed consent and underwent PH screening. Baseline data were recorded on ethnicity, Scadding stage on chest radiography [12], pulmonary function test and chest computed tomography. $\mathrm{PH}$ screening consisted of thorough history taking, physical examination and echocardiography by the same experienced physician. Based on the European Society of Cardiology/European Respiratory Society guideline for PH [2], patients were divided into three groups: 1) low $\mathrm{PH}$ probability, with maximal tricuspid regurgitation velocity $\left(\mathrm{TRV}_{\max }\right)$ absent or $\leqslant 2.8 \mathrm{~m} \cdot \mathrm{s}^{-1}$ without secondary $\mathrm{PH}$ signs; 2 ) intermediate $\mathrm{PH}$ probability, with $\mathrm{TRV}_{\max }$ absent or $\leqslant 2.8 \mathrm{~m} \cdot \mathrm{s}^{-1}$ with secondary $\mathrm{PH}$ signs, or $2.9-3.4 \mathrm{~m} \cdot \mathrm{s}^{-1}$ without secondary PH signs; and 3) high $\mathrm{PH}$ probability, with $\mathrm{TRV}_{\max } 2.9-3.4 \mathrm{~m} \cdot \mathrm{s}^{-1}$ with secondary $\mathrm{PH}$ signs, or $\mathrm{TRV}_{\max }>3.4 \mathrm{~m} \cdot \mathrm{s}^{-1}$.

Secondary PH signs were divided into three groups in accordance to the guideline: ventricles, pulmonary artery, and right atrium. Secondary signs were counted as present if one or more secondary PH signs of at least two different secondary sign groups were present. All patients with intermediate or high probability were referred for RHC. Presence of $\mathrm{PH}$ was defined as a mean pulmonary artery pressure of $\geqslant 25 \mathrm{mmHg}$. Patients with $\mathrm{PH}$ were discussed in a multidisciplinary $\mathrm{PH}$ team for the final diagnosis. Patients with a low $\mathrm{PH}$ probability with minor secondary signs for $\mathrm{PH}$ (defined as only one secondary sign or two secondary signs from the same group) were re-evaluated after 1 year. In case of progression, patients were still referred for RHC.

@ERSpublications

The prevalence of pulmonary hypertension in sarcoidosis seems to differ between ethnicities and was never investigated in a Caucasian cohort. This study shows a prevalence of $3 \%$ in a Caucasian cohort, significantly lower compared to other ethnicities. http://bit.ly/2kRMrap

Cite this article as: Huitema MP, Bakker ALM, Mager JJ, et al. Prevalence of pulmonary hypertension in pulmonary sarcoidosis: the first large European prospective study. Eur Respir J 2019; 54: 1900897 [https:// doi.org/10.1183/13993003.00897-2019]. 
As shown in figure 1, 512 patients were eligible for inclusion, of whom 399 patients signed informed consent and underwent PH screening (57.9\% male, mean \pm SD age $49.4 \pm 11.6$ years). The main ethnicity was Caucasian (90.5\%). Patients had a mean \pm SD history of sarcoidosis for $6.0 \pm 8.2$ years, and $20 \%$ had Scadding stage IV sarcoidosis. Low, intermediate and high $\mathrm{PH}$ probability was present in $92.2 \%, 5.5 \%$ and $1.5 \%$, respectively. High or intermediate $\mathrm{PH}$ probability was present in 28 patients, of whom four refused RHC. Of the low $\mathrm{PH}$ probability group and inconclusive echocardiograms, four patients underwent RHC based on clinical judgement, of whom none had PH. In total, 28 patients underwent RHC. In 10 out of these 28 patients, $\mathrm{PH}$ was present. One patient had a post-capillary component.

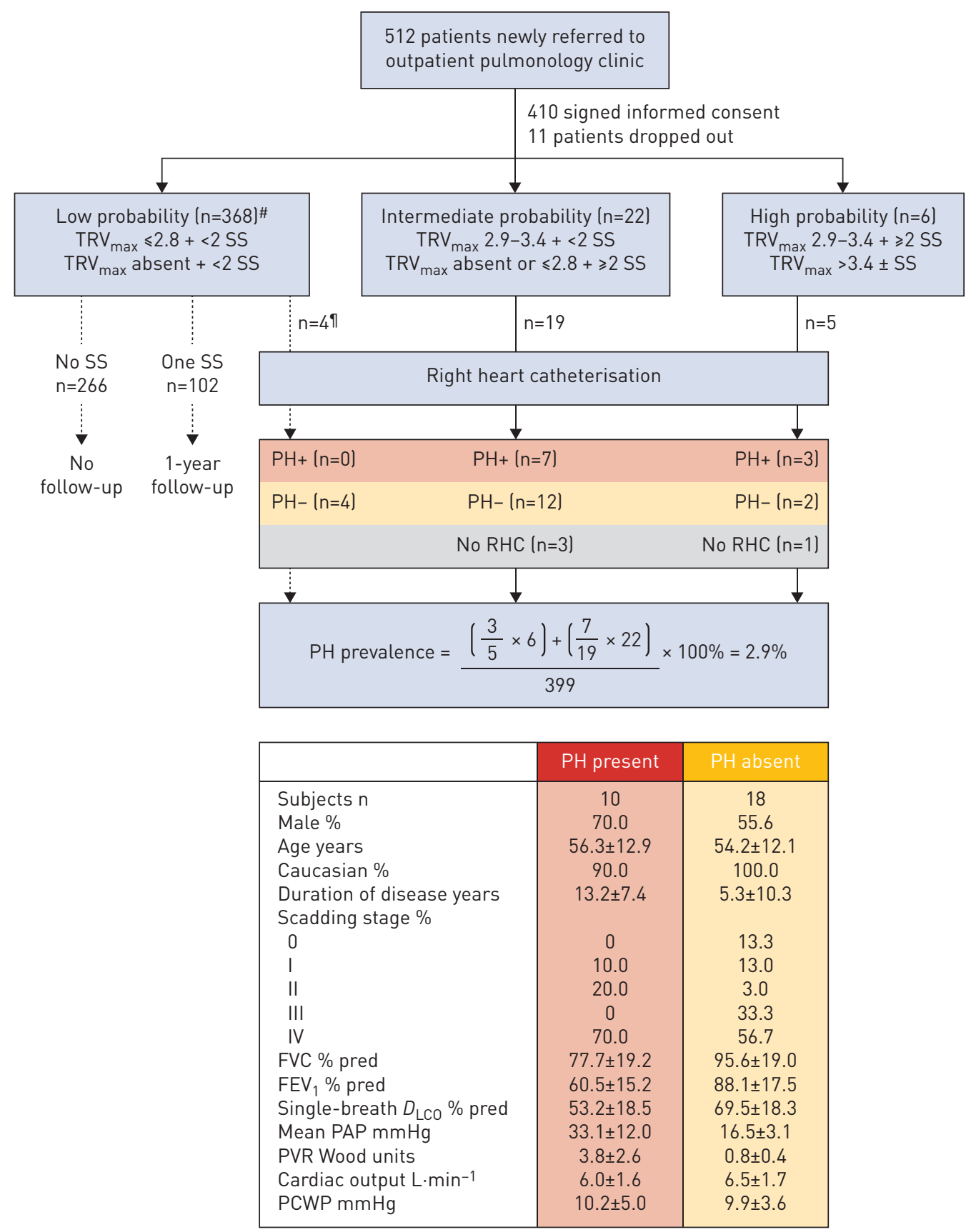

FIGURE 1 Flow chart for pulmonary hypertension $(\mathrm{PH})$ screening, including outcomes for patients with $\mathrm{PH}$ present compared to $\mathrm{PH}$ absent. $\mathrm{TRV}_{\max }$ : maximal tricuspid regurgitation velocity; SS: secondary signs; RHC: right heart catheterisation; FVC: forced vital capacity; $\mathrm{FEV}_{1}$ : forced expiratory volume in $1 \mathrm{~s} ; D_{\mathrm{Lco}}$ : diffusing capacity of the lung for carbon monoxide; PAP: pulmonary artery pressure; PVR: pulmonary vascular resistance; PCWP: pulmonary capillary wedge pressure. \#: three patients had an inconclusive echocardiogram; ": none of the four patients with low PH probability undergoing RHC had PH. Data are presented as mean $\pm \mathrm{SD}$, unless otherwise stated. 
As shown in figure 1, patients with $\mathrm{PH}$ had a longer history of sarcoidosis and presented with Scadding stage IV more often. Three out of the 10 patients developed PH in the absence of significant fibrosis.

Based on the results of echocardiography and RHC, the PH prevalence was calculated. Because a few patients with high and intermediate $\mathrm{PH}$ probability did not undergo RHC, the fraction with $\mathrm{PH}$ of the patients who underwent RHC within either one of the $\mathrm{PH}$ probability groups was multiplied by the total number of patients of the same $\mathrm{PH}$ probability group. The estimated $\mathrm{PH}$ prevalence in this cohort of sarcoidosis patients is $2.9 \%$. This number could range from $2.5 \%$ (if all of the missing RHCs in the high and intermediate probability groups ruled out $\mathrm{PH}$ ) up to $3.5 \%$ (if all confirmed $\mathrm{PH}$ ).

Of the low $\mathrm{PH}$ probability group, 102 patients had minor secondary signs. 98 underwent re-evaluation after 1 year. Only two patients showed progression of the secondary signs, in one of whom $\mathrm{PH}$ was ruled out by RHC. The other patient had developed a severe cardiomyopathy due to cardiac sarcoidosis with a subsequent post-capillary $\mathrm{PH}$.

The PULSAR study is the first large study investigating the PH prevalence in a predominantly Caucasian cohort of almost 400 consecutive sarcoidosis patients referred to a tertiary sarcoidosis centre using echocardiography and, if indicated, RHC. As a result, the $\mathrm{PH}$ prevalence is estimated to be around $3 \%$. Three studies previously investigated the $\mathrm{PH}$ prevalence in sarcoidosis independently of symptoms or signs for PH. HANDA et al. [6] were the first, investigating 246 consecutive Japanese sarcoidosis patients visiting the outpatient clinic for follow-up, defining $\mathrm{PH}$ as an RVSP of $\geqslant 40 \mathrm{mmHg}$ on echocardiography. They found an echocardiographic PH prevalence of 5.7\%. BOURBONNAIS and SAMAVATI [7] prospectively evaluated 141 sarcoidosis patients with echocardiography, followed by RHC in 35 patients. PH was defined as an RVSP of $40 \mathrm{mmHg}$ in the absence of significant left heart dysfunction. RHC was performed in these patients, and also in patients with inconclusive echocardiography with repeatedly abnormal 6-min walk test outcomes despite optimisation of therapy. They found a $\mathrm{PH}$ prevalence of $14 \%$. 88\% were African American descendants, who are more likely to have sarcoidosis-associated PH compared to Caucasians [13]. In a third study, AlHamad et al. [8] investigated 96 Arab sarcoidosis patients, defining PH as an RVSP of $\geqslant 40 \mathrm{mmHg}$ as measured by echocardiography. A prevalence of $20.8 \%$ was reported, with a predominance of female $\mathrm{PH}$ patients. The prevalence of $3 \%$ in our population is significantly lower. This might be due to a less biased and well defined study population. Furthermore, prevalence of $\mathrm{PH}$ might differ between ethnicities.

Although this study presents the largest cohort of sarcoidosis patients prospectively investigated for $\mathrm{PH}$, there are several limitations. First, not all patients underwent the gold standard RHC due to ethical considerations. We acknowledge that echocardiography might not always rule out $\mathrm{PH}$ correctly in patients with low PH probability. In clinical practice, the decision to perform RHC should outweigh the potential risks. Secondly, we aimed to minimise selection bias; however, some bias could not be avoided, since patients with worse disease severity are more likely to be referred to a tertiary centre. Finally, $62 \%$ of all patients were on immunosuppressive therapy at baseline, which might influence the haemodynamic profile at the moment of screening.

In conclusion, the $\mathrm{PH}$ prevalence is estimated to be around 3\% in a cohort of predominantly Caucasian sarcoidosis patients referred to a Dutch tertiary centre. It can be suggested that there are ethnic differences in the prevalence of $\mathrm{PH}$.

Marloes P. Huitema ${ }^{1}$, Annelies L.M. Bakker ${ }^{1}$, Johannes J. Mager ${ }^{2}$, Benno J.W.M. Rensing ${ }^{1}$, Fokko Smits ${ }^{3}$, Repke J. Snijder ${ }^{2}$, Jan C. Grutters ${ }^{2,4}$ and Marco C. Post ${ }^{1}$

${ }^{1}$ Dept of Cardiology, St Antonius Hospital, Nieuwegein, The Netherlands. ${ }^{2}$ Dept of Pulmonology, St Antonius Hospital, Nieuwegein, The Netherlands. ${ }^{3}$ Dept of Radiology, St Antonius Hospital, Nieuwegein, The Netherlands. ${ }^{4}$ Dept of Pulmonology, University Medical Centre Utrecht, Utrecht, The Netherlands.

Correspondence: Marloes P. Huitema, Dept of Cardiology, St Antonius Hospital, Koekoekslaan 1, Nieuwegein, 3435 CM, The Netherlands. E-mail: mp.huitema@antoniusziekenhuis.nl

Received: 29 Nov 2018 | Accepted after revision: 12 June 2019

Conflict of interest: None declared.

Support statement: Funding was received from ZonMw (The Netherlands Organisation for Health Research and Development). Funding information for this article has been deposited with the Crossref Funder Registry.

\section{References}

Spagnolo P, Rossi G, Trisolini R, et al. Pulmonary sarcoidosis. Lancet Respir Med 2018; 6: 389-402.

Galiè N, Humbert M, Vachiery JL, et al. 2015 ESC/ERS Guidelines for the diagnosis and treatment of pulmonary hypertension: The Joint Task Force for the Diagnosis and Treatment of Pulmonary Hypertension of the European Society of Cardiology (ESC) and the European Respiratory Society (ERS). Eur Respir J 2015; 46: 903-975. 
3 Nunes H, Humbert M, Capron F, et al. Pulmonary hypertension associated with sarcoidosis: mechanisms, haemodynamics and prognosis. Thorax 2006; 61: 68-74.

4 Boucly A, Cottin V, Nunes $\mathrm{H}$, et al. Management and long-term outcomes of sarcoidosis-associated pulmonary hypertension. Eur Respir J 2017; 50: 1700465.

5 Zimmerman I, Mann N. Boeck's sarcoid; a case of sarcoidosis complicated by pulmonary emphysema and cor pulmonale. Ann Intern Med 1949; 31: 153-162.

6 Handa T, Nagai S, Miki S, et al. Incidence of pulmonary hypertension and its clinical relevance in patients with sarcoidosis. Chest 2006; 129: 1246-1252.

7 Bourbonnais JM, Samavati L. Clinical predictors of pulmonary hypertension in sarcoidosis. Eur Respir J 2008; 32: 296-302.

8 Alhamad EH, Idrees MM, Alanezi MO, et al. Sarcoidosis-associated pulmonary hypertension: clinical features and outcomes in Arab patients. Ann Thorac Med 2010; 5: 86-91.

9 Baughman RP, Engel PJ, Taylor L, et al. Survival in sarcoidosis-associated pulmonary hypertension: the importance of hemodynamic evaluation. Chest 2010; 138: 1078-1085.

10 Milman N, Svendsen CB, Iversen M, et al. Sarcoidosis-associated pulmonary hypertension: acute vasoresponsiveness to inhaled nitric oxide and the relation to long-term effect of sildenafil. Eur Respir J 2009; 3: 207-213.

11 Rizzato G, Pezzano A, Sala G, et al. Right heart impairment in sarcoidosis: haemodynamic and echocardiographic study. Eur J Respir Dis 1983; 64: 121-128.

12 Scadding JG. Prognosis of intrathoracic sarcoidosis in England. A review of 136 cases after five years' observation. Br Med J 1961; 2: 1165-1172.

13 Mirsaeidi M, Machado RF, Schraufnagel D, et al. Racial difference in sarcoidosis mortality in the United States. Chest 2015; 147: 438-449. 